\title{
Remarks on the Hall Conductivity in Chiral Superconductors: Weak vs. Strong Coupling Approach
}

\author{
M. ROGATKO AND K.I. WYSOKIŃSKI* \\ Institute of Physics, M. Curie-Skłodowska University, I. Radziszewskiego 10, PL-20-031 Lublin, Poland

\begin{abstract}
We discuss the calculations of the ac Hall conductivity for superconductors with the time reversal symmetry breaking states. In the weak coupling theories these states show vanishing Hall response in one band models, even though one expects otherwise on symmetry grounds. On the other hand, the strong coupling approach based on the anti-de Sitter-conformal field theory correspondence leads to the non-vanishing Hall conductivity. We discuss the possible reasons of the discrepancy. The weak coupling many orbital theory leading to the Hall conductivity with correct temperature dependence is also briefly presented.
\end{abstract}

DOI: 10.12693 /APhysPolA.126.A-9

PACS: $74.20 .-\mathrm{z}, 74.25 . \mathrm{F}-$, 74.70.Pq, 11.25.Tq

\section{Introduction}

Among spin singlet and spin triplet superconductors there exist states, allowed by the group theoretical analysis which break time reversal symmetry (TRS) [1]. The known example of such state is the triplet chiral state with $\boldsymbol{d}$-vector

$$
\boldsymbol{d}(\boldsymbol{k})=\Delta(T)\left[\sin \left(k_{x} a\right) \pm \mathrm{i} \sin \left(k_{y} a\right)\right] \hat{e}_{z},
$$

which is realized in the A-phase of ${ }^{3} \mathrm{He}[2]$ and most probably in strontium ruthenate $\left(\mathrm{Sr}_{2} \mathrm{RuO}_{4}\right)[3,4]$, even though the singlet time reversal symmetry breaking state $\Delta(\boldsymbol{k})=\Delta(T)\left[\sin \left(k_{x} a\right)+\mathrm{i} \sin \left(k_{y} a\right)\right] \sin \left(k_{z} c\right)$ has also been proposed [5] as providing an alternative explanation of phase sensitive experiments [6] in $\mathrm{Sr}_{2} \mathrm{RuO}_{4}$. Similar time reversal symmetry breaking singlet state $\Delta(\boldsymbol{k})=$ $\Delta_{1}(T)\left[\cos \left(k_{x} a\right)-\cos \left(k_{y} a\right)\right]+\mathrm{i} \sin \left(k_{x} a\right) \sin \left(k_{y} a\right)$ has been recently proposed as a possible ground state in the bi layer silicene [7].

The above mentioned states all break time reversal symmetry and are expected to lead to nonzero value of the ac Hall conductivity, which can be measured by means of e.g. the Kerr effect. The effect has indeed been measured in strontium ruthenate with high accuracy [8]. Careful calculations, however, indicate that in one band systems the Hall conductivity vanishes, unless one takes e.g. collective mode response or impurity scattering into account. The situation seem to be different in the strong coupling approach. By strong coupling we understand here the approach based on the holographic description of superconductivity as proposed recently [9].

In the rest of the paper we shall briefly discuss weak and strong coupling approaches to calculations of the Hall conductivity of the chiral superconductors paying special attention to the apparent discrepancy: the vanishing of the Hall conductivity in weak coupling one band theories

${ }^{*}$ corresponding author; e-mail: karol@tytan.umcs.lublin.pl and its appearance in the holographic models (i.e. at strong coupling). We also give a brief account of the novel approach based on many orbital description of superconductors, which leads to the Hall conductivity in the weak coupling.

\section{Absence of ac Hall conductivity in one band weak coupling theories}

As a preparation to the subsequent symmetry analysis we start with the derivation of the Hall conductivity formula which is given by the paramagnetic current $\boldsymbol{j}_{p}(\boldsymbol{r})$ response to the electromagnetic field. The diamagnetic contribution is diagonal and does not contribute to the Hall conductivity. The field is described by the position $r$ and time $t$ dependent vector potential $\boldsymbol{A}(\boldsymbol{r}, t)$. The minimal coupling between the charged system and the electromagnetic field is given by [10] $H^{\prime}=-e \int \mathrm{d} \boldsymbol{r} \boldsymbol{j}_{p}(\boldsymbol{r}) \boldsymbol{A}(\boldsymbol{r}, t)$. In the linear response limit the current in the system averaged over the appropriate ensemble is to the linear order in perturbation given by

$$
\begin{aligned}
& \left\langle\boldsymbol{j}_{\alpha}(\boldsymbol{r}, t)\right\rangle=-e \sum_{\beta} \int \mathrm{d} \boldsymbol{r}^{\prime} \int_{-\infty}^{+\infty} \mathrm{d} t^{\prime} \\
& \quad \times K_{\alpha, \beta}\left(\boldsymbol{r}, \boldsymbol{r}^{\prime}, t-t^{\prime}\right) A_{\beta}\left(\boldsymbol{r}^{\prime}, t^{\prime}\right),
\end{aligned}
$$

where the retarded response function $K_{\alpha, \beta}\left(\boldsymbol{r}, \boldsymbol{r}^{\prime}, t-t^{\prime}\right)=$ $-\frac{\mathrm{i}}{\hbar} \Theta\left(t-t^{\prime}\right)\left\langle\left[j_{p \alpha}\left(\boldsymbol{r}, t-t^{\prime}\right), j_{p \beta}\left(\boldsymbol{r}^{\prime}, 0\right)\right]\right\rangle$ and the symbol $\langle\cdots\rangle$ denotes averaging over the equilibrium distribution function defined for the unperturbed system, i.e. at times before the perturbation has been turned on and [...] is a commutator.

In a homogeneous superconducting system with the order parameter $\Delta$ and for periodic perturbation $\boldsymbol{A}(\boldsymbol{r}, t)=$ $\boldsymbol{A}(\boldsymbol{k}, \omega) \mathrm{e}^{\mathrm{i}(\boldsymbol{k} \boldsymbol{r}-\omega t)}+$ c.c. the physical current $\boldsymbol{J}(\boldsymbol{k}, \omega)=$ $e \boldsymbol{j}(\boldsymbol{k}, \omega)$ may be written in terms of the Fourier components of $\boldsymbol{A}(\boldsymbol{k}, \omega)$ as

$$
J_{\alpha}(\boldsymbol{q}, \omega)=\sum_{\beta} K_{\alpha, \beta}(\boldsymbol{k}, \omega, \Delta) A_{\beta}(\boldsymbol{k}, \omega),
$$

where we used the notation of Yip and Sauls [11], which 
is convenient to study symmetry properties of the system. The kernel is defined as

$$
\begin{aligned}
& K_{\alpha, \beta}(\boldsymbol{k}, \omega, \Delta)=-\frac{\mathrm{i}}{\hbar} \int_{-\infty}^{+\infty} \mathrm{d} t \int \mathrm{d} \boldsymbol{r} \mathrm{e}^{\mathrm{i}(\omega t-\boldsymbol{k} \boldsymbol{r})} \\
& \quad \times \Theta(t)\left\langle\left[j_{p \alpha}(\boldsymbol{r}, t), j_{p \beta}(0,0)\right]\right\rangle .
\end{aligned}
$$

The symmetry analysis helps to find the necessary condition for the Hall effect to exist. First we are dealing with tetragonal system possessing four-fold symmetry axis along $c$-axis of the crystal. This leads to the constraints on the tensor $K_{\alpha, \beta}: K_{x x}=K_{y y}$ and $K_{y x}=-K_{x y}$. To proceed it is convenient to rewrite Eq. (3) in terms of $J_{ \pm}=J_{x} \pm \mathrm{i} J_{y}$ and $E_{ \pm}=E_{x} \pm \mathrm{i} E_{y}$ in the diagonal form

$$
J_{ \pm}(\boldsymbol{k}, \omega)=K_{ \pm}(\boldsymbol{k}, \omega, \Delta) E_{ \pm}(\boldsymbol{k}, \omega),
$$

where $K_{ \pm}=K_{x x} \pm \mathrm{i} K_{y x}$. The necessary but not sufficient condition, as will be discussed later, for the appearance of the Hall conductivity is that $K_{+} \neq K_{-}$.

The additional symmetries which have to be taken into account are the two-dimensional parity $P$ with respect to $x$ - or $y$-axis (being the operation $x \rightarrow-x$ or $y \rightarrow-y$ ), time reversal symmetry $T$ and causality. In the present context the causality is the property that the response function $K\left(\boldsymbol{r}, t-t^{\prime}\right)$ is non-zero for $t>t^{\prime}$ only. In analogy to [11] we find that causality, $P$ and $T$ symmetries lead to the following properties of the kernel $K_{ \pm}(\boldsymbol{k}, \omega, \Delta)$ defined in (4) $[12,13]$ :

$$
\begin{aligned}
& {\left[K_{+}(\boldsymbol{k}, \omega, \Delta)\right]^{*}=K_{-}(-\boldsymbol{k},-\omega, \Delta), \text { causality, }} \\
& K_{+}(\boldsymbol{k}, \omega, \Delta)=K_{-}\left(\boldsymbol{k}, \omega, \Delta^{P}\right), \text { parity, } \\
& K_{+}(\boldsymbol{k}, \omega, \Delta)=K_{-}\left(-\boldsymbol{k}, \omega, \Delta^{T}\right), \text { time reversal. }
\end{aligned}
$$

In the above formulae symbols $\Delta^{P, T}$ denote the states obtained from $\Delta$ by the corresponding symmetry operation. It is easily seen that if the system is invariant under the time reversal operation i.e. $\Delta^{T}=\Delta$ then in the long-wave-length limit $\boldsymbol{k}=0$ one finds $K_{+}=K_{-}$and no Hall conductivity in the system. On the other hand, it follows from (parity) that the breaking of parity is necessary condition for the existence of Hall conductivity.

\section{AdS/CFT correspondence and chiral superconductors with Hall response}

Recently the interesting new direction of research has been started, which proposes to use methods of the theory of gravity to solve some problems of condensed matter physics [14]. Among notable examples is the metalsuperconductor phase transition $[15,16]$. The correspondence has been established [17] between scale invariant conformal field theory (CFT) in D-dimensions without gravity with the version of $(\mathrm{D}+1)$-dimensional theory of gravity (for a popular presentation see [18]). It is the version of string theory with anti-de Sitter space-time (AdS and hence AdS/CFT or gauge/gravity correspondence). The apparent advantage of using the string theory formalism is that the strong coupling problems in the former (CFT) appear as weak coupling in the later (gravity) theory [19]. The temperature is brought by the black hole in the bulk.
To describe the superconductor with $s$-wave order parameter the authors [9] assumed the existence of the black hole described by $\mathrm{d} s^{2}=-f(r) \mathrm{d} t^{2}+\frac{\mathrm{d} r^{2}}{f(r)}+r^{2}\left(\mathrm{~d} x^{2}+\mathrm{d} y^{2}\right)$, with $f(r)=\frac{r^{2}}{L^{2}}-\frac{M}{r}$ and $L$ being the AdS radius. $M$ and $L$ determine the Hawking temperature of the aforementioned black hole $T=\frac{3 M^{1 / 3}}{4 \pi L^{4 / 3}}$. The superconducting condensate is described by the charged complex field $\Psi(r)$ in analogy to phenomenological Ginzburg-Landau approach to the superconductivity. In the matter Lagrangian density $\mathcal{L}$ besides this complex field also the electromagnetic field (Maxwell) is taken into account. In the units with all constants taken as unity the Lagrangian density is written as

$$
\mathcal{L}=-\frac{1}{4} F^{a b} F_{a b}+\frac{2|\Psi|^{2}}{L^{2}}-|\partial \Psi-\mathrm{i} A \Psi|^{2} .
$$

Its variation over all fields (components of the electromagnetic field and complex scalar field) lead to field equations. The boundary solutions have the properties of an $s$-wave strongly coupled superconductor with $2 \Delta(0) / k_{\mathrm{B}} T_{c} \approx 8$. The solution also shows infinite zero frequency conductivity.

There exist proposals of the holographic superconductors with other symmetries: $p, p+\mathrm{i} p, d, d+\mathrm{i} d$, etc. For our discussion the time reversal symmetry breaking order parameters $p+\mathrm{i} p$ and $d+\mathrm{i} d$ are of special interest, as they could give non-vanishing Hall conductivity without an external magnetic field. The modeling of the $d$-wave superconductor in the gravity theory, however, is demanding as the correct Lagrangian of the spin 2 field in the curved background is not known. One way of obtaining the $d$-wave condensate is to consider charged spin 2 field in the asymptotical AdS geometry. Although the spin 2 field Lagrangian in curved background is not known, the authors [20] claimed that they elaborate the one which had limits with the Fierz-Pauli Lagrangian in flat spacetime for neutral case and with Federbush one, for a charged spin two field. The holographic model for massive charged spin 2 field and the fermionic field proposed by the authors [20] contains terms describing interaction of the massive field with the gravity background. The obtained solution shows condensation of the spin 2 field below critical temperature. At the same time the spectral function corresponding to the fermion field shows the Fermi arcs reminiscent to that seen in photoemission spectroscopy of $d$-wave cuprate superconductors.

The generalization of the paper [20] to $d+\mathrm{i} d$ chiral symmetry has also been proposed [21]. These authors used the same Lagrangian with spin 2 field and fermionic field. Studying the underlying problem in the probe limit (no back-reaction on the metric) they reproduced the results of the previous authors and calculated conductivity tensor. Their calculations indicate that the frequency dependent Hall conductivity is non-zero in the chiral condensed state. This constitutes the remarkable result in contradiction to weak coupling theories. This difference calls for detailed understanding of the result. 


\section{Weak vs. strong coupling: towards resolution of the discrepancy}

In a single band situation the crucial symmetry is the particle-hole one. As it has been discussed in [11] the perfect particle-hole symmetry in a single band model implies that the state of the system is identical to its time reversed counterpart, which leads to vanishing Hall conductivity. It turns out that in models with many non-degenerate bands the particle-hole symmetry is not even approximate symmetry and does not forbid existence of $\sigma_{x y}$. The microscopic calculations show $[22,23]$ that even in such models the Hall conductivity vanishes in states like (1) which have $\eta_{y}^{m}=\mathrm{i} \eta_{x}^{m}$ for each orbital $m$. As we have seen the non-vanishing Hall response can be obtained in many orbital models with more general states (10).

On the other hand, as already mentioned there are suggestions [21] that holographic analogy leads to non-zero Hall conductivity $\operatorname{Im} \sigma_{x y}(\omega)$ for states of $d+\mathrm{i} d$ character without that property. The obvious discrepancy requires the explanation. One plausible argument is that the gauge-gravity duality takes vertex corrections into account and breaks particle-hole symmetry due to the presence of gravitational field. Another one is related to the spontaneous orbital momentum generation in gravity theory. The recent paper [24] suggests that in gravity models the breaking of the parity induces orbital momentum. This general property of the gauge/gravity duality could serve as a plausible explanation of the discrepancy. The issue, however, is more intriguing because the orbital momentum have also been predicted and calculated $[25,26]$ in the weak coupling approach to chiral finite size system. The orbital momentum here are in fact finite size effects with similar temperature dependence to the bulk orbital momentum appearing in many orbital theory [27], but strongly depending on the boundary conditions. The orbital momentum of the bulk system is directly related to the Hall conductivity [28] by the so-called dichroic sum-rule [29].

In any case the exhaustive understanding of the mechanism of Hall conductivity generation in holographic theories is necessary before attempts to the more thorough modeling of the superconducting systems will be started.

\section{Hall response in many orbital models}

Early calculations of the Kerr effect [30] $\left(\propto \sigma_{H}(\omega)\right)$ in chiral superconductors were done in the context of $p_{x} \pm \mathrm{i} p_{y}$ state proposed to be realized in heavy fermion materials [11]. These authors performed careful symmetry analysis and their microscopic calculations show that for the existence of the Hall effect in the system the TRS and two-dimensional parity $(P)$ breaking is not enough and one needs collective mode response (i.e. vertex corrections) and the particle Hall asymmetry.

It seems to be a general rule that the TRS and $P$ breaking is a necessary but not sufficient condition for the appearance of the Hall effect. For example in the normal metals the existence of the Hall effect requires magnetic field (external or internal) and spin-orbit coupling [31]. The same is true in the superconducting state. The Hall conductivity vanishes in one-band models with chiral order parameter (1). Technically this cancellation results from the fact that the calculations require summation of the response over the whole Brillouin zone of the crystal and the contributions from different parts of it cancel each other [22].

At present there are two theoretical approaches which give Hall conductivity in the time reversal symmetry breaking state in strontium ruthenate. One of them invokes scattering on impurities $[32,33]$ and thus may be termed an extrinsic mechanism. Technically it relies on the impurity induced vertex corrections to the currentcurrent correlation function. The other $[34,28]$ relies on the many orbital description of the low energy states. It operates in systems with the Fermi surfaces consisting of many pieces.

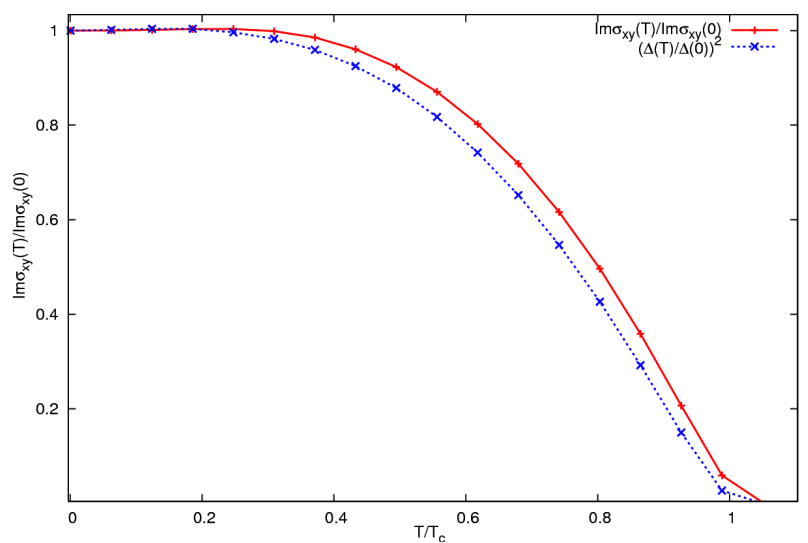

Fig. 1. Typical temperature dependence of the Hall conductivity and $\Delta(T)^{2}$ in the chiral three-orbital superconductor at constant frequency $\omega$.

In Fig. 1 we show the imaginary part of the frequency dependent Hall conductivity $\operatorname{Im} \sigma_{x y}(\omega, T)$ normalized to its zero temperature value $\operatorname{Im} \sigma_{x y}(\omega, 0)$ plotted as a function of temperature in units of superconducting transition temperature $T / T_{c}$. It is worth noting that the temperature dependence shown in the figure is similar to $T$-dependence of the Kerr effect [28] measured for $\mathrm{Sr}_{2} \mathrm{RuO}_{4}$ [8].

In this approach the essential ingredient of the theory $[28,23]$ is the orbital dependent spatial symmetry of the chiral order parameter

$$
\boldsymbol{d}_{\nu}(\boldsymbol{k})=\left[\eta_{x}^{\nu} \sin \left(k_{x} a\right)+\eta_{y}^{\nu} \sin \left(k_{y} a\right)\right] \hat{e}_{z} .
$$

The symmetry properties of the order parameters in two orbitals $\nu$ denoted by $a$ and $b$ read

$$
\eta_{y}^{a}=\mathrm{i} \eta_{x}^{b} \text { and } \eta_{y}^{b}=\mathrm{i} \eta_{x}^{a} \text {. }
$$

These symmetries guarantee the chiral character of the superconducting state, while the relation $\left|\eta_{x}^{\nu}\right| \neq\left|\eta_{y}^{\nu}\right|$, $\nu=a, b$ is crucial for the Hall effect to exist as discussed recently [23]. This relation makes the symmetry of each 
of the orbital dependent states lower than the symmetry of the system. On the other hand, full symmetry is restored [23] if one takes symmetries (11) into account. The calculation shown in figure were done for the same model of strontium ruthenate as described earlier [23].

\section{Concluding remarks}

In summary we have pointed out the discrepancy between the calculations of the Hall conductivity for chiral superconductors within the standard weak coupling (condensed matter) and strong coupling (holographic) approaches. In the standard approach the existence of the effect requires order parameter collective response or impurity scattering while it seems to appear in a gravity approach as a result of spontaneous momentum generation, albeit the details of the discrepancy are not clear at present. They will be a subject of future studies.

In the Kubo linear response theory the Hall effect appears naturally in chiral superconductors if many hybridised orbitals exist near the Fermi energy. The obtained temperature dependence (shown in Fig. 1) agrees with that measured by the Kerr effect [8].

\section{References}

[1] V.P. Mineev, K.V. Samokhin, Introduction to Unconventional Superconductivity, Gordon and Breach Science, Amsterdam 1999.

[2] A.J. Leggett, Rev. Mod. Phys. 47, 331 (1975).

[3] A.P. Mackenzie, Y. Maeno, Rev. Mod. Phys. 75, 657 (2003).

[4] C. Kallin, Rep. Prog. Phys. 75, 042501 (2012).

[5] I. Zutic, I. Mazin, Phys. Rev. Lett. 95, 217004 (2005).

[6] K.D. Nelson, Z.Q. Mao, Y. Maeno, Y. Liu, Science 306, 1151 (2004).

[7] Feng Liu, Cheng-Cheng Liu, Kehui Wu, Fan Yang, Yugui Yao, Phys. Rev. Lett. 111, 066804 (2013).

[8] Jing Xia, Y. Maeno, P.T. Beyersdorf, M.M. Fejer, A. Kapitulnik, Phys. Rev. Lett. 97, 167002 (2006).

[9] S.A. Hartnoll, C.P. Herzog, G.T. Horowitz, Phys. Rev. Lett. 101, 031601 (2008).

[10] M. di Ventra, Electrical Transport in Nanoscale Systems, Cambridge Univ. Press, Cambridge 2008.
[11] S.K. Yip, J.A. Sauls, J. Low. Temp. Phys. 86, 257 (1992).

[12] D.N. Zubariew, Termodynamika statystyczna, PWN, Warszawa 1974 (in Polish).

[13] R. Kubo, M. Toda, N. Hashitsume, Fizyka statystyczna, PWN, Warszawa 1991, (in Polish).

[14] S.A. Hartnoll, Class. Quant. Grav. 26, 224002 (2009).

[15] C.P. Herzog, J. Phys. A Math. Theor. 42, 343001 (2009).

[16] G.T. Horowitz, Class. Quantum Grav. 28, 114008 (2011).

[17] J.M. Maldacena, Adv. Theor. Math. Phys. 2, 231 (1998).

[18] J.M. Maldacena, Sci. Am. 293(5), 24 (2005) [see also Świat Nauki 172(12), 30 (2005), in Polish].

[19] S. Sachdev, Ann. Rev. Cond. Matter Phys. 3, 9 (2012).

[20] F. Benini, C.P. Herzog, A. Yarom, Phys. Lett. B 701, 626 (2011).

[21] Jiunn-Wei Chen, Yu-Sheng Liu, D. Maity, J. High Energy Phys. 5, 32 (2011).

[22] V.P. Mineev, J. Phys. Soc. Jpn. 81, 093703 (2012).

[23] M. Gradhand, K.I. Wysokinski, J.F. Annett, B.L. Györffy, Phys. Rev. B 88, 094504 (2013).

[24] Hong Liu, H. Ooguri, B. Stoica, N. Yunes, Phys. Rev Lett. 110, 211601 (2013).

[25] M. Stone, R. Roy, Phys. Rev. B 69, 184511 (2004).

[26] J.A. Sauls, Phys. Rev. B 84, 214509 (2011).

[27] J.F. Annett, B.L. Gyorffy, K.I. Wysokinski, New J. Phys. 11, 055063 (2009).

[28] K.I. Wysokinski, J.F. Annett, B.L. Györffy, Phys Rev. Lett. 108, 077004 (2012).

[29] I. Souza, D. Vanderbilt, Phys. Rev. B 77, 054438 (2008).

[30] H.S. Bennett, E.A. Stern, Phys. Rev. 137, A488 (1965).

[31] H. Ebert, Rep. Prog. Phys. 59, 1665 (1996).

[32] J. Goryo, Phys. Rev. B 78, 060501 (2008).

[33] R.M. Lutchyn, P. Nagornykh, V.M. Yakovenko, Phys. Rev. B 80, 104508 (2009).

[34] E. Taylor, C. Kallin, Phys. Rev. Lett. 108, 157001 (2012). 